\title{
Short-term pretreatment with a dual 5 $\alpha$-reductase inhibitor before bipolar transurethral resection of the prostate (B-TURP): evaluation of prostate vascularity and decreased surgical blood loss in large prostates
}

\author{
Gian Maria Busetto, Riccardo Giovannone, Gabriele Antonini, Antonella Rossi*, \\ Francesco Del Giudice, Stefano Tricarico, Giulia Ragonesi, Vincenzo Gentile and \\ Ettore De Berardinis
}

Department of Gynecological-Obstetrics Sciences and Urological Sciences, Policlinico Umberto I Sapienza Rome University, and *Department of Pathology, Tor Vergata University, Rome, Italy

\section{Objective}

To investigate if short-term treatment with dutasteride ( 8 weeks) before bipolar transurethral resection of the prostate (B-TURP) can reduce intraoperative bleeding, as dutasteride a dual $5 \alpha$-reductase inhibitor (5-ARI) blocks the conversion of testosterone into its active form dihydrotestosterone (DHT), and reduces prostate volume and prostate-specific antigen (PSA) levels, while increasing urinary flow rate.

\section{Patients and Methods}

In all, 259 patients were enrolled and randomised to two groups: Group A, receiving placebo and Group B, receiving dutasteride ( $0.5 \mathrm{mg}$ daily for 8 weeks). Blood samples were taken before and after B-TURP for serum chemistry evaluation. In particular we evaluated blood parameters associated with blood loss [haemoglobin ( $\mathrm{Hb})$ and haematocrit (Ht)] and prostate vascularity [vascular endothelial growth factor (VEGF) immunoreactivity and microvessel density (MVD) using cluster of differentiation 34 (CD34) immunoreactivity].

\section{Results}

Total testosterone, DHT, PSA level and prostate volume were evaluated and with the exception of DHT and PSA level there was no statistically significant differences between the groups. When comparing changes in $\mathrm{Hb}$ and $\mathrm{Ht}$ between Group A and Group B before and after B-TURP, there was a statistically significant difference only in patients with large prostates of $\geq 50 \mathrm{~mL}$ ( $\Delta \mathrm{Hb} 3.86$ vs $2.05 \mathrm{~g} / \mathrm{dL}$ and $\Delta \mathrm{Ht} 4.98$ vs $2.64 \%$, in Groups A and B, respectively). There was no significant difference in MVD and VEGF index in prostates of $<50 \mathrm{~mL}$, conversely in large prostates the difference become statistically significant.

\section{Conclusions}

Dutasteride was able to reduce operative and perioperative bleeding only in patients with large prostates $(\geq 50 \mathrm{~mL})$ that underwent B-TURP. Our findings are confirmed by $\mathrm{Hb}$ and $\mathrm{Ht}$ values reported before and after the B-TURP and reductions in the molecular markers for VEGF and CD34 in the dutasteride-treated specimens.

\section{Keywords}

dutasteride, B-TURP, bleeding

\section{Introduction}

Benign prostatic enlargement and the histologically confirmed diagnosis of $\mathrm{BPH}$ is the most important cause of LUTS. BPH has an incidence of up to $40 \%$ in men in their fifth decade and increases with age [1].
The nuclear-bound steroid enzyme $5 \alpha$-reductase, primarily in the prostatic stroma cells, converts testosterone into its active form, dihydrotestosterone (DHT). Two isoforms of this enzyme are present in the human body: type 1 mainly expressed in the liver and skin and type 2 mainly expressed and active in the prostate. $5 \alpha$-reductase inhibitors (5-ARIs) 
block this conversion and have the ability to reduce prostate volume by $18-28 \%$, reduce PSA levels by $50 \%$, increase urinary flow and decrease the incidence of urinary retention [2,3]. 5-ARIs should be offered to patients with moderate-to-severe $\mathrm{BPH}$ and with an enlarged prostate $(>40 \mathrm{~mL})$ to prevent disease progression. 5-ARIs, through their action, decrease the activity of androgen-controlled growth factors finally responsible for angiogenesis and theoretically reduce operative prostatic bleeding [4]. In particular finasteride decreases vascular endothelial growth factor (VEGF) expression as well as microvessel density (MVD) in prostatic tissue [5].

Dutasteride is a 5-ARI that acts specifically on both isotypes of $5 \alpha$-reductase (type 1 and 2); finasteride, on the other hand, is selective for isoenzyme type 2 . Dutasteride, in comparison with finasteride, is a 45-fold greater inhibitor of type 1 and 2.5 -fold greater inhibitor of type $25 \alpha$-reductase [6,7]. Dutasteride achieves a serum DHT reduction of $>90 \%$, whereas finasteride results in a suppression of up to $70 \%$ [8]. Considering the better DHT suppression by dutasteride, we speculated that it may also have a greater effect on vascularisation. Ku et al. [9] reported that in the prostate of rats, treated with at least 4 weeks dutasteride, there is lower expression of VEGF and hypoxia-inducible factor $1 \alpha$ (HIF-1 $\alpha$ ), two main factors correlated with vascularisation.

5-ARIs can be offered to men with moderate-to-severe LUTS with an enlarged prostate to prevent disease progression but due to the slow onset of action are suitable only for long-term treatment (many years).

In accordance with European Association of Urology guidelines, TURP remains the 'gold standard' surgical treatment for LUTS secondary to BPH in prostates with a volume of 30-80 mL. TURP can decrease most symptoms, with improvement in all urodynamic parameters and is superior to medical therapy [10]. One of the main intra- and perioperative complications is haemorrhage that can lead to bladder clot retention and an incidence of blood transfusion of up to $8.4 \%$ [11]. Bipolar TURP (B-TURP), with the introduction of plasmakinetic bipolar technology, represents an important improvement of the traditional TURP. Monopolar TURP vs B-TURP is reported to have almost the same efficacy, while the overall incidence of side-effects is lower for B-TURP (28.6\% vs $15.5 \%)$ [12]. The main advantages are less bleeding, with reduced fluid absorption, reduction of clot retention with lower blood transfusion rate and virtual elimination of transurethral resection syndrome due to the use of saline irrigation.

Assuming that dutasteride reduces prostatic vascularisation, and considering B-TURP as the 'gold standard' treatment in patients with BPH with LUTS, we investigated if a short-term treatment with dutasteride ( 8 weeks) before B-TURP could reduce intraoperative bleeding. We also evaluated VEGF and cluster of differentiation 34 (CD34), an immunohistochemical marker of the MVD, in resected prostatic tissue.

\section{Patients and Methods}

Between April 2011 and May 2013, 259 patients with a mean (range) age of $68(60-81)$ years were randomised to receive daily $0.5 \mathrm{mg}$ dutasteride or placebo for 8 weeks before B-TURP. This was a single-centre study and all patients were referred to the Department of Urology, Policlinico Umberto I Sapienza Rome University. Men in Group A received placebo pretreatment and those in Group B received dutasteride ( $0.5 \mathrm{mg}$ daily for 8 weeks) in a 1:1 ratio. For better evaluation of the results, randomisation was stratified by prostate volume $(<50$ and $\geq 50 \mathrm{~mL})$. All the patients enrolled were treated before B-TURP with $\alpha$-blockers. Every patient, the day before B-TURP, underwent a TRUS of the prostate to evaluate the exact volume of the gland. Moreover, $1 \mathrm{~h}$ before B-TURP, blood was drawn for serum chemistry evaluation. Patients taking NSAIDs, anticoagulant or antiplatelet drugs discontinued 2 weeks before B-TURP. All surgical procedures were carried out under spinal anaesthesia, the surgeon used a 24-F resectoscope (Karl Storz, Tuttlingen, Germany) at low pressure with continuous flow and at the end a three-way $18-22 \mathrm{~F}$ catheter was placed. Intravenous hydration was started at the beginning of anaesthesia $(1000 \mathrm{~mL})$ and was continued for the day of the procedure and maintained constant at $1500 \mathrm{~mL}(100 \mathrm{~mL} / \mathrm{h})$. At $48 \mathrm{~h}$ after B-TURP a second blood sample was drawn.

Exclusion criteria included: patients with renal function impairment (blood creatinine $>145 \mu \mathrm{mol} / \mathrm{L}$ ) due to its effect on blood clotting; patients with an alteration of coagulation parameters [international normalised ratio (INR) $>1.3$ ] or any kind of bleeding disorders; patients that, in the past, had already undergone any kind of prostate surgery; patients that had already assumed a different scheme, period or dosage of any kind of 5-ARI; refusal to sign the informed consent. Considering the aforementioned criteria, 19 patients were excluded.

For the study outcomes the following parameters were evaluated: haemoglobin $(\mathrm{Hb})$ and haematocrit $(\mathrm{Ht})$ before and after B-TURP, PSA level, total testosterone (TT) and DHT levels, and we also calculated resected prostate volume and B-TURP duration. Resected prostate chips were histologically analysed ensuring separation from those extracted from the urethra. Afterwards, the material was stained with monoclonal antibodies directed against CD34 and VEGF. In particular, a CD34 value and a VEGF index were obtained. Tissue specimens, fixed in $10 \%$ buffered formalin and embedded in paraffin, were stained with haematoxylin and eosin. Sections were cut and slides were dewaxed in xylene and rehydrated through a graded series of ethanols. Heat-induced epitope retrieval was carried out by immersing the slides in citrate 
buffer (pH 6) and microwaving at $600 \mathrm{~W}$ for 20 min before rinsing with PBS. Endogenous peroxidase activity was quenched by incubating the sections in $1 \%$ hydrogen peroxide. Nonspecific binding sites were then blocked by pre-incubating with $20 \%$ normal serum and $1 \%$ BSA in PBS/0.3\% Triton X-100 (Union Carbide, Dow Corporation, Midland, MI, USA) for $20 \mathrm{~min}$ at room temperature. Sections were incubated with polyclonal rabbit anti-VEGF antibody at a concentration of 1:50, as well as monoclonal murine anti-CD34 at a concentration of 1:20. After washing with $0.25 \%$ BSA and $0.05 \%$ polysorbate 20 in PBS, sections were incubated with biotinylated secondary pan-specific antibody at 1:500 for $1 \mathrm{~h}$ at room temperature. Sections were again washed in PBS with $0.05 \%$ polysorbate 20 , then incubated with horseradish peroxidase and conjugated streptavidin-biotin complex for $45 \mathrm{~min}$. All sections were again washed in PBS with $0.05 \%$ polysorbate 20 . Immunoreactivity was then visualised by adding hydrogen peroxide as an enzyme substrate, in the presence of $0.05 \% 3,3^{\prime}$-diaminobenzidine. Nuclei were then lightly counterstained with Harris's haematoxylin. All the areas of most intense neovascularisation were evaluated by scanning at low magnification $(\times 10-100)$, avoiding areas with lymphocytic infiltration or fibrosis, and seven to eight sections for each patient were included (Figs 1,2). Any brown-staining endothelial cell (CD34-positive) containing a visible nucleus and clearly separate from adjacent microvessels, epithelial cells, and other connective tissue elements, was considered a single, countable microvessel, without requirement for a lumen or the presence of erythrocytes. The microvessels were counted in a $0.74 \mathrm{~mm}^{2}$ area. VEGF immunoreactivity was scored for the percentage of stained epithelial glandular and endothelial cells, as $0,1+, 2+$, or $3+$, according to staining intensity [13]. A hypertrophic area with any degree of staining was scored as VEGF-positive. Hyperplastic areas that were VEGF-positive and VEGF-negative were assigned the score of the area with strongest staining.

Spontaneously reported adverse events or those noted by the investigator were recorded throughout the study.

Ethical committee approval was obtained and all treatments applied are part of routine standard care; the registration number is 21 and was obtained on 7th April 2014. The study was conducted in line with European Urology and Good Clinical Practice guidelines, with ethical principles laid down in the latest version of the Declaration of Helsinki. Every patient signed an informed consent to participate in the study.

Statistical analysis was carried out with BMDP statistical software, version 7 (Statistical Solutions, Saugus, MA, USA) and SPSS (Chicago, IL, USA; version 15.00 for Windows). For all analyses, we used two-sided tests, with $P<0.05$ considered to indicate statistical significance.

Continuous variables are reported as the mean (SD) and compared between groups using Student's $t$-test for
Fig. 1 Placebo group - immunohistochemical evaluation of prostatic tissue with higher expression of MVD (CD34) and brown stained spot representing vessels, $\times 100$.

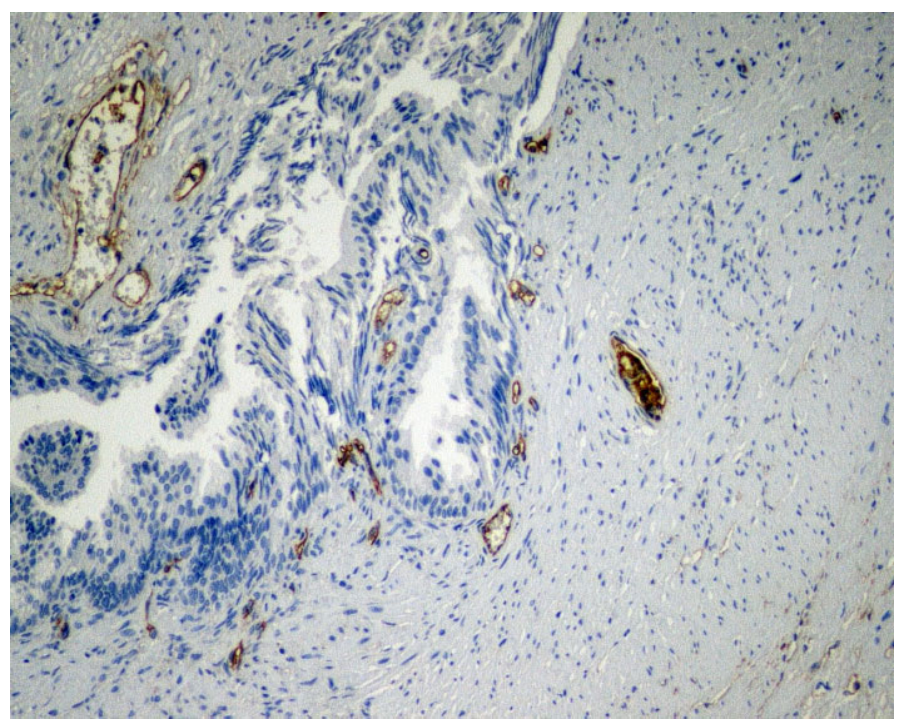

Fig. 2 Dutasteride group - immunohistochemical evaluation of prostatic tissue with lower expression of MVD (CD34) and brown stained spot representing vessels, $\times 100$

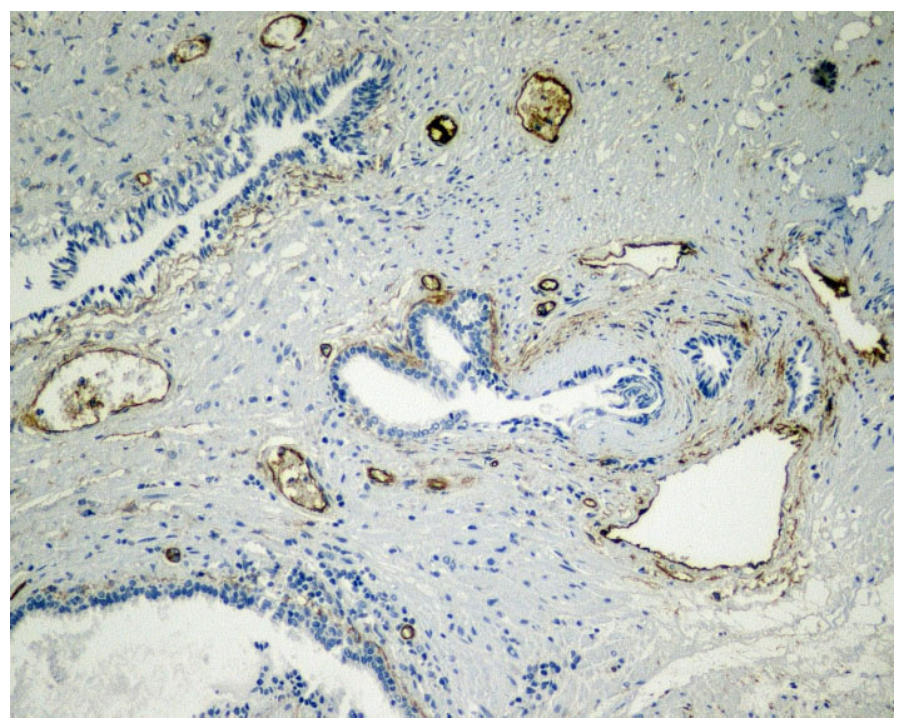

independent samples or using the Welch-corrected $t$-test in case of heteroscedasticity. Categorical variables are reported as the number of occurrences and percentages.

Analysis of covariance (ANCOVA) was used to assess the significance of variation in $\mathrm{Hb}$ and $\mathrm{Ht}$ levels before and after B-TURP. In this analysis, Group (placebo vs dutasteride) was used as fixed factor and preoperative values of outcome variables were entered as covariates. Results of ANCOVA models 
are expressed in terms of estimated marginal means with 95\% CIs.

A post hoc power analysis showed that a sample size of 120 per group (for each level of the stratification factor) would allow the detection of a medium effect size (Cohen's $d=0.5$ ) with a power of $80 \%$ using a two-tailed $t$-test and a significance level of 0.05 .

\section{Results}

Of the 259 enrolled patients, 19 did not meet inclusion criteria. Both Group A (placebo pretreatment) and Group B (dutasteride pretreatment) consisted each of 120 eligible patients. TT, DHT, PSA levels and prostate volume were evaluated and with the exclusion of DHT and PSA levels there were no statistically significant differences between the groups (Table 1). All patients underwent B-TURP and the mean (SD) operative time was 50 (10) min in Group A and 48 (13) min in Group B $(P=0.23)$. After B-TURP the volume removed was evaluated and was, respectively, 31.58 (9.87) $\mathrm{mL}$ in Group $\mathrm{A}$ and 32.15 (8.36) $\mathrm{mL}$ in Group B $(P=0.88)$.

Table 1 The patients' characteristics.

\begin{tabular}{lccr}
\hline Variable & $\begin{array}{c}\text { Group A } \\
\text { placebo }\end{array}$ & $\begin{array}{c}\text { Group B } \\
\text { dutasteride }\end{array}$ & $\boldsymbol{P}$ \\
\hline Patients, $n$ & 120 & 120 & \\
Mean (range) age, years & $67(61-78)$ & $68(60-81)$ & 0.9 \\
Mean (SD): & & & \\
Body mass index, kg/m ${ }^{2}$ & $27.34(8.5)$ & $28.12(7.6)$ & 0.21 \\
PSA level, ng/mL & $4.48(3.2)$ & $2.33(1.15)$ & $<0.05$ \\
TT, ng/dL & $525(284)$ & $588(335)$ & 0.34 \\
DHT, ng/dL & $54(33)$ & $5(4)$ & $<0.05$ \\
Prostate volume, mL & $60.35(35.86)$ & $61.15(34.73)$ & 0.79 \\
Volume removed, $\mathrm{mL}$ & $31.58(9.87)$ & $32.15(8.36)$ & 0.88 \\
Operating time, min & $50(10)$ & $48(13)$ & 0.23 \\
\hline
\end{tabular}

$\mathrm{Hb}$ and $\mathrm{Ht}$ values, evaluated $1 \mathrm{~h}$ before and $48 \mathrm{~h}$ after the B-TURP, were correlated between Group A and B in accordance with prostate size, and in particular we compared prostates with a volume $<50 \mathrm{~mL}$ and those with a volume $\geq 50 \mathrm{~mL}$. Considering small prostates $(<50 \mathrm{~mL})$ and according to the ANCOVA results, the reduction in $\mathrm{Hb}$ values from before to after B-TURP was $2.41 \mathrm{~g} / \mathrm{dL}$ (95\% CI 1.39-3.43) in Group A and $1.92 \mathrm{~g} / \mathrm{dL}$ (95\% CI 1.00-2.84) in Group B (difference between groups: $0.49 \mathrm{~g} / \mathrm{dL}, 95 \% \mathrm{CI}-0.26$ to $1.24 ; P=0.454)$. A decrease in Ht levels was also seen both in the placebo group (2.22\%; 95\% CI 1.77-2.67) and the dutasteride group (1.95\%; 95\% CI 1.50-2.40) without a statistically significant treatment effect (difference between groups: $-0.27 \%$; $95 \% \mathrm{CI}-0.91$ to $0.38 ; P=0.411)$.

In men with prostates $\geq 50 \mathrm{~mL}$ the reduction in $\mathrm{Hb}$ was significantly lower in Group B (2.05 g/dL; 95\% CI 1.47-2.61) than in Group A (3.86 g/dL; 95\% CI 3.29-4.43) with a treatment effect equal to $-1.81 \mathrm{~g} / \mathrm{dL}(95 \% \mathrm{CI}-2.62$ to -1.0 ; $P<0.05)$; Group B was also characterised by a significantly lower reduction in Ht levels vs Group A (2.64\% vs $4.98 \%$; difference between groups: $-2.34 \%$; $95 \% \mathrm{CI}-3.51$ to -1.17 ; $P<0.05)$.

The mean (SD) MVD and VEGF index in prostates of $<50 \mathrm{~mL}$ were 18.64 (1.12) and 2.09 (0.48) in Group A and 17.04 (0.40) and $1.73(0.36)$ in Group B respectively, without a statistically significant difference. The mean (SD) MVD and VEGF index in prostates of $\geq 50 \mathrm{~mL}$ were 24.17 (1.80) and 6.73 (0.95) in Group A and 19.49 (0.44) and 2.44 (0.49) in Group B (both $P<0.05)$. All results are listed in Table 2.

\section{Discussion}

Benign prostatic enlargement, caused by hyperplasia of both stromal and acinar cells in the periurethral transition zone, is also characterised by an increase in vascularisation.

Table 2 Blood parameters and vascularisation in prostates of $<50$ and $\geq 50 \mathrm{~mL}$.

\begin{tabular}{|c|c|c|c|c|c|c|}
\hline \multirow[t]{2}{*}{ Variable } & \multicolumn{3}{|c|}{ Prostates <50 mL } & \multicolumn{3}{|c|}{ Prostates $\geq 50 \mathrm{~mL}$} \\
\hline & $\begin{array}{l}\text { Group A } \\
\text { placebo }\end{array}$ & $\begin{array}{c}\text { Group B } \\
\text { dutasteride }\end{array}$ & $\boldsymbol{P}$ & $\begin{array}{l}\text { Group A } \\
\text { placebo }\end{array}$ & $\begin{array}{c}\text { Group B } \\
\text { dutasteride }\end{array}$ & $\boldsymbol{P}$ \\
\hline Patients, $n$ & 39 & 38 & & 81 & 82 & \\
\hline \multicolumn{7}{|l|}{ Mean (SD) Hb, g/dL: } \\
\hline before B-TURP & $14.37(2.12)$ & $13.92(2.12)$ & 0.48 & $14.30(2.10)$ & $14.01(2.08)$ & 0.54 \\
\hline after B-TURP & $12.34(1.86)$ & $12.19(2.12)$ & 0.35 & $10.50(2.49)$ & $11.91(2.15)$ & $<0.05$ \\
\hline$\Delta \mathrm{Hb}, \mathrm{g} / \mathrm{dL}(95 \% \mathrm{CI})^{*}$ & $2.41(1.39-3.43)$ & $1.92(1.00-2.84)$ & 0.45 & $3.86(3.29-4.43)$ & $2.05(1.47-2.61)$ & $<0.05$ \\
\hline \multicolumn{7}{|l|}{ Mean (SD) Ht, \%: } \\
\hline before B-TURP & $43.15(3.20)$ & $42.48(4.23)$ & 0.67 & $43.00(3.20)$ & $42.66(4.15)$ & 0.60 \\
\hline after B-TURP, \% & $40.78(3.01)$ & $40.15(4.77)$ & 0.57 & $38.20(3.84)$ & $40.16(3.24)$ & $<0.05$ \\
\hline$\Delta \mathrm{Ht}, \%(95 \% \mathrm{CI})^{*}$ & $2.22(1.77-2.67)$ & $1.95(1.50-2.40)$ & 0.41 & $4.98(4.16-4.80)$ & $2.64(1.82-3.46)$ & $<0.05$ \\
\hline \multicolumn{7}{|l|}{ Mean (SD): } \\
\hline MVD & $18.64(1.12)$ & $17.04(0.40)$ & 0.09 & $24.17(1.80)$ & $19.49(0.44)$ & $<0.05$ \\
\hline VEGF index & $2.09(0.48)$ & $1.73(0.36)$ & 0.09 & $6.73(0.95)$ & $2.44(0.49)$ & $<0.05$ \\
\hline
\end{tabular}


Following our previous experience with finasteride, used as a pretreatment to reduce bleeding during monopolar TURP, we decided to evaluate if dutasteride, a theoretically more effective drug, was able to achieve better results. Furthermore, differing from the previous trial we used the latest surgical technique, B-TURP [14].

Considering the results of our present trial we can conclude that a short pretreatment with dutasteride is able to reduce B-TURP bleeding but only when treating large prostates with a volume of $\geq 50 \mathrm{~mL}$. In particular, in this subgroup of patients, there is a statistically significant difference in $\Delta \mathrm{Hb}$ and $\Delta \mathrm{Ht}$ (3.86 vs $2.05 \mathrm{~g} / \mathrm{dL}$ and 4.98 vs $2.64 \%$, respectively), which favours dutasteride-treated patients. The present results are confirmed by CD34 and VEGF evaluations.

Looking at the blood parameters of the patients in both groups, the first consideration is that 8 weeks of dutasteride is able to reduce DHT by $\approx 90 \%$ and have only a slight action on TT (not statistically significant). When comparing the DHT values after pretreatment with finasteride, it is evident that dutasteride pretreatment achieves a larger reduction and this is correlated with the capability of the first to inhibit only $5 \alpha$-reductase type 2 , while the second has an action on both types (1 and 2). Amory et al. [15] reported that dutasteride and finasteride reduce DHT by $94 \%$ and $73 \%$, respectively, with transiently increased testosterone.

Although the clinical role of dual inhibition remains unclear, it is established that 5-ARIs induce apoptosis of prostate epithelial cells leading to gland volume reduction and PSA level decreases [16]. Analysing the PSA levels after 8 weeks of therapy with the 5-ARI, we found that there was a reduction of $\approx 48 \%$ compared with the placebo group. In the literature it is reported that 5-ARIs are able to reduce PSA levels by $\approx 50 \%[2,3]$. Prostate volume, the volume removed and operative time were about the same for the two groups. It has been reported that 5-ARIs are able to reduce LUTS by $15-30 \%$, decrease prostate volume by $18-28 \%$ and increase maximum urinary flow rate by $1.5-2.0 \mathrm{~mL} / \mathrm{s}$, but only after a long period of treatment (2-4 years) [10]. Furthermore, 5 -ARIs reduce the risk of acute urinary retention or need for surgery; in particular dutasteride decreases the risk of acute urinary retention by $57 \%$ and surgical intervention by $48 \%$ [17].

To explain why 5-ARIs should be able to reduce prostate bleeding during surgery and recovery, we have to look to the decrease in blood vessel number that accompanies the shrinking of the prostate [18]. This conclusion can also be extrapolated from many studies reporting a significant decrease in gross haematuria secondary to benign prostatic enlargement $[19,20]$. However, when looking at specific studies on decreased bleeding during endoscopic surgery, there are conflicting results.
Focusing on trials using dutasteride as the 5-ARI, Boccon-Gibod et al. [21], in a multicentre study with 59 patients randomised to receive 4 weeks of daily placebo or $0.5 \mathrm{mg}$ dutasteride, reported no significant difference in bleeding between groups (1.4 vs $1.9 \mathrm{Hb} / \mathrm{g}$ resected tissue). In the experience of Hahn et al. [22], there was no significant reduction in bleeding between patients receiving a treatment before surgery with $2-4$ weeks dutasteride vs placebo. On the other hand, Kravchick et al. [23] reported that 6 weeks dutasteride treatment can reduce, in the periurethral area proximal to the verumontanum, prostate tissue vascularity. Also the Martov et al. [24] study, which included 70 patients with large prostates $(>80 \mathrm{~mL})$ treated with $\geq 1$ month dutasteride before TURP, noted a reduction in blood loss and operative time together with an increase in removed tissue. Pastore et al. [25], from their experience, suggested that pretreatment with dutasteride for 6 weeks could considerably reduce bleeding during TURP.

The results of our present trial are novel and are an important contribution to the understanding of the ability of 5-ARIs to reduce bleeding during endoscopic surgery. Firstly, our present trial represents a step forward because is it one of the few considering dutasteride as the 5-ARI (dual inhibition) and also because we analysed a large number of patients (240 randomised patients). Furthermore, we are the first to find a direct correlation between bleeding and the size of the prostate adenoma. Focusing on prostate size, there are only a few published papers stressing the relation between the size and the effect against LUTS: finasteride is effective only when treating prostates $>40 \mathrm{~mL}$ and dutasteride is also efficacious when considering a volume of $30 \mathrm{~mL}$ [26,27]. In particular, in order to categorise and to make our findings more homogeneous, we decided to divide our groups according to prostate volume. Considering prostates with a volume of $<50 \mathrm{~mL}$ we noted no statistically significant difference in $\mathrm{Hb}$ and $\mathrm{Ht}$ between the placebo- and dutasteride-treated groups; conversely, when considering large prostates $(\geq 50 \mathrm{~mL})$, there was a statistically significant difference in the reduction in blood counts when comparing the treatment and placebo groups.

To evaluate angiogenesis and vascularisation of the prostate, we evaluated VEGF, which stimulates proliferation and migration of endothelial cells playing a role in physiological and pathological angiogenesis, and CD34, a glycosylated type I transmembrane protein expressed in the luminal surface of most endothelial cells of small vessels. Zaitsu et al. [28] in their paper, report that dutasteride is able to reduce the vascularisation of the prostate. In particular, they analysed prostate sections stained with haematoxylin/eosin and anti-CD31 antibody, finding that artery/arteriole density and vein/venule density in benign prostatic tissue were lower in the 5-ARI-treated group. Pareek et al. [29] conducted a study to elucidate the mechanism by which finasteride reduces 
prostatic urethral bleeding and concluded that decreased expression of VEGF inhibits angiogenesis and significantly decreases MVD in prostatic suburethral tissue.

Our present results are also divided by the size of the prostate because we again noted a correlation with the volume of the gland. Considering prostates with a volume of $<50 \mathrm{~mL}$, the VEGF index and CD34 values reported do not differ in a statistically significant way between patients treated with placebo or 5-ARI. When looking at prostates with a volume of $\geq 50 \mathrm{~mL}$, our present findings confirm a statistically significant difference in the VEGF index and CD34 values between the two groups.

Although this is a randomised study, there is some potential biases that could represent some limitations: patient's physical features, such as coagulation and body mass index, histological analysis of the resected tissue, surgical duration and quality.

In conclusion, dutasteride, $0.5 \mathrm{mg}$ daily for 8 weeks, is able to reduce operative and perioperative bleeding in patients submitted to B-TURP only if a large prostate $(\geq 50 \mathrm{~mL})$ is being treated. Our findings are confirmed by $\mathrm{Hb}$ and $\mathrm{Ht}$ values reported before and after the B-TURP and by reductions in the molecular markers for VEGF and CD34 in the dutasteride-treated specimens.

\section{Conflict of Interest}

None declared.

\section{References}

1 Berry SJ, Coffey DS, Walsh PC, Ewing LL. The development of human benign prostatic hyperplasia with age. J Urol 1984; 132: 474-9

2 Marks LS, Partin AW, Dorey FJ et al. Long-term effects of finasteride on prostate tissue composition. Urology 1999; 53: 574-80

3 Naslund MJ, Miner M. A review of the clinical efficacy and safety of 5a-reductase inhibitors for the enlarged prostate. Clin Ther 2007; 29: $17-25$

4 Puchner PJ, Miller MI. The effects of finasteride on hematuria associated with benign prostatic hyperplasia: a preliminary report. J Urol 1995; 154: 1779-82

5 Pareek G, Shevchuk M, Armenakas NA et al. The effect of finasteride on the expression of vascular endothelial growth factor and microvessel density: a possible mechanism for decreases prostatic bleeding in treated patients. J Urol 2003; 169: 20-3

6 Marberger M. Drug insight: $5 \alpha$-reductase inhibitors for the treatment of benign prostatic hyperplasia. Nat Clin Pract Urol 2006; 3: 495-503

7 Frye SV. Discovery and clinical development of dutasteride, a potent dual 5 $\alpha$-reductase inhibitor. Curr Top Med Chem 2006; 6: 405-21

8 Roehrborn CG, Andriole G, Schalken JA, Wilson T, Clark R. Dutasteride, a novel dual 5-alpha reductase inhibitor, reduces serum DHT to a greater extent versus finasteride and achieves finasteride maximal reduction in a larger proportion of patients. Eur Urol Suppl 2003; 2: 161, Abstract 635

9 Ku JH, Shin JK, Cho MC, Myung JK, Moon KC, Paick JS. Effect of dutasteride on the expression of hypoxia-inducible factor-1alpha, vascular endothelial growth factor and microvessel density in rat and human tissue. Scand J Urol Nephrol 2009; 43: 445-53
10 Oelke M, Bachmann A, Descazeaud A et al. EAU guidelines on the treatment and follow-up of non-neurogenic male lower urinary tract symptoms including benign prostatic obstruction. Eur Urol 2013; 64: $118-40$

11 Madersbacher S, Marberger M. Is transurethral resection of the prostate still justified? Br J Urol 1999; 83: 227-37

12 Issa MM. Technological advances in transurethral resection of the prostate: bipolar versus monopolar TURP. J Endourol 2008; 22: 1587-95

13 Toi M, Hoshina S, Takayanagi T, Tominaga T. Association of vascular endothelial growth factor expression with tumor angiogenesis and with early relapse in primary breast cancer. Cancer Sci 1994; 85: 1045-9

14 De Berardinis E, Antonini G, Busetto GM, Gentile V, Di Silverio F, Rossi A. Reduced intraoperative bleeding during transurethral resection of the prostate: evaluation of finasteride, vascular endothelial growth factor, and CD34. Curr Prostate Rep 2008; 6: 123-7

15 Amory JK, Wang C, Swerdloff RS et al. The effect of 5alpha-reductase inhibition with dutasteride and finasteride on semen parameters and serum hormones in healthy men. J Clin Endocrinol Metab 2007; 92: 1659-65

16 Rittmaster RS, Norman RW, Thomas LN, Rowden G. Evidence for atrophy and apoptosis in the prostates of men given finasteride. J Clin Endocrinol Metab 1996; 81: 814-9

17 Roehrborn CG, Boyle P, Nickel JC et al. Efficacy and safety of dual inhibitors of 5-alpha-reductase types 1 and 2 (dutasteride) in men with benign prostatic hyperplasia. Urology 2002; 60: 434-41

18 Lekas E, Bergh A, Damber JE. Effects of finasteride and bicalutamide on prostatic blood flow in the rat. BJU Int 2000; 85: 962-5

19 Carlin BI, Bodner DR, Spirnak JP, Resnick MI. Role of finasteride in the treatment of recurrent hematuria secondary to benign prostatic hyperplasia. Prostate 1997; 31: 180-2

20 Delakas D, Lianos E, Karyotis I, Cranidis A. Finasteride: a long-term follow-up in the treatment of recurrent hematuria associated with benign prostatic hyperplasia. Urol Int 2001; 67: 69-72

21 Boccon-Gibod L, Valton M, Ibrahim H, Boccon-Gibod L, Comenducci A. Effect of dutasteride on reduction of intraoperative bleeding related to transurethral resection of the prostate. Prog Urol 2005; 15: 1085-9

22 Hahn RG, Fagerström T, Tammela TL et al. Blood loss and postoperative complications associated with transurethral resection of the prostate after pretreatment with dutasteride. BJU Int 2007; 99: 587-94

23 Kravchick S, Cytron S, Mamonov A, Peled R, Linov L. Effect of short-term dutasteride therapy on prostate vascularity in patients with benign prostatic hyperplasia: a pilot study. Urology 2009; 73: $1274-8$

24 Martov AG, Ergacov DV. The experience in dutasteride use before transurethral prostatic resection for large adenoma. Urologiia 2008; 46 : $48-52$

25 Pastore A, Mariani S, Barrese F et al. Transurethral resection of prostate and the role of pharmacological treatment with dutasteride in decreasing surgical blood loss. J Endourol 2013; 27: 68-70

26 Boyle P, Gould AL, Roehrborn CG. Prostate volume predicts outcome of treatment of benign prostatic hyperplasia with finasteride: meta-analysis of randomized clinical trials. Urology 1996; 48: 398-405

27 Roehrborn CG, Lukkarinen O, Mark S, Siami P, Ramsdell J, Zinner N. Long-term sustained improvement in symptoms of benign prostatic hyperplasia with the dual $5 \alpha$-reductase inhibitor dutasteride: results of 4-years studies. BJU Int 2005; 96: 572-7

28 Zaitsu M, Tonooka A, Mikami $\mathrm{K}$ et al. A dual $5 \alpha$-reductase inhibitor dutasteride caused reductions in vascular density and area in benign prostatic hyperplasia. ISRN Urol 2013; 2013: 1-8

29 Pareek G, Shevchuk M, Armenakas NA et al. The effect of finasteride on the expression of vascular endothelial growth factor and microvessel 
density: a possible mechanism for decreased prostatic bleeding in treated patients. J Urol 2003; 169: 20-3

Correspondence: Ettore De Berardinis, Policlinico Umberto I, Sapienza Rome University, viale del Policlinico 155, 00161 Rome, Italy.

e-mail: ettore.deberardinis@uniroma1.it
Abbreviations: 5-ARI, $5 \alpha$-reductase inhibitor; B-TURP, bipolar TURP; CD34, cluster of differentiation 34; DHT, dihydrotestosterone; $\mathrm{Hb}$, haemoglobin; $\mathrm{Ht}$, haematocrit; MVD, microvessel density; TT, total testosterone; VEGF, vascular endothelial growth factor. 